This item was submitted to Loughborough's Research Repository by the author.

Items in Figshare are protected by copyright, with all rights reserved, unless otherwise indicated.

\title{
System fault diagnostics using fault tree analysis
}

PLEASE CITE THE PUBLISHED VERSION

PUBLISHER

(C) IMechE / Professional Engineering Publishing

LICENCE

CC BY-NC-ND 4.0

REPOSITORY RECORD

Hurdle, Emma E., L.M. Bartlett, and J.D. Andrews. 2008. "System Fault Diagnostics Using Fault Tree Analysis". figshare. https://hdl.handle.net/2134/3817. 
This item was submitted to Loughborough's Institutional Repository (https://dspace.lboro.ac.uk/) by the author and is made available under the following Creative Commons Licence conditions.

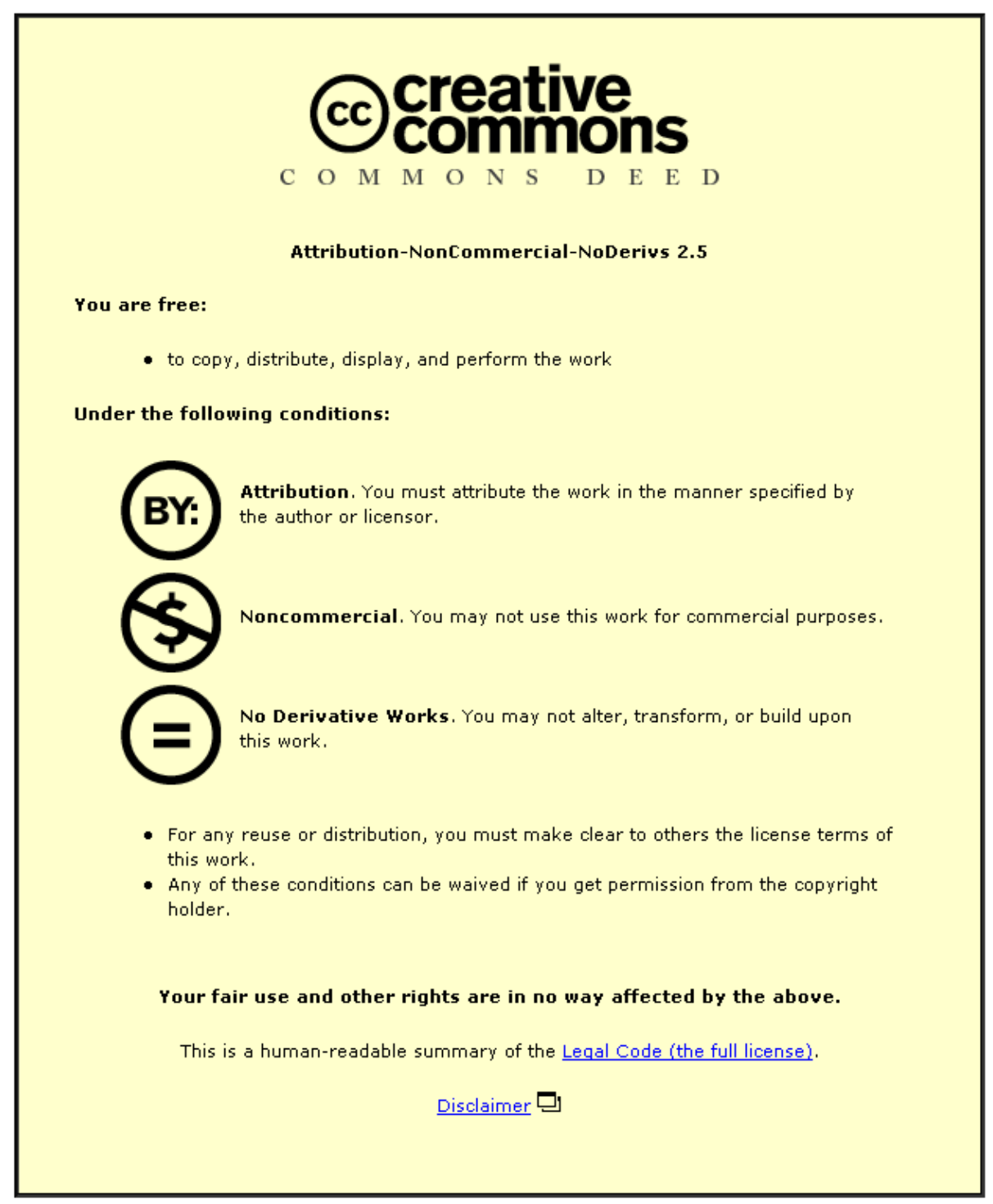

For the full text of this licence, please go to: http://creativecommons.org/licenses/by-nc-nd/2.5/ 


\title{
System fault diagnostics using fault tree analysis
}

E E Hurdle, L M Bartlett, and J D Andrews*

Department of Aeronautical and Automotive Engineering, Loughborough University, Loughborough, UK

The manuscript was received on 15 June 2005 and was accepted after revision for publication on 17 May 2006.

DOI: $10.1243 / 1748006 X J R R 6$

\begin{abstract}
Over the last 50 years, advances in technology have led to an increase in the complexity and sophistication of systems. More complex systems can be harder to maintain and the root cause of a fault more difficult to isolate.

Downtime resultin from a system failure can be dangerous or expensive, depending on the type of system. In aircraft systems the ability to diagnose quickly the causes of a fault can have a significant impact on the time taken to rectify the problem and to return the aircraft to service. In chemical prcess plants the need to diagnose causes of a safety-critical failure in a system can be vital and a diagnosis may be required within minutes. Speed of fault isolation can save time, reduce costs, and increase company productivity and therefore profits. System fault diagnosis is the process of identifying the cause of a malfunction by observing its effect at various test points.

Fault tree analysis (FTA) is a method that describes all possible causes of a specified system state in terms of the state of the components within the system. A system model is used to identify the states that the system should be in at any point in time. This paper presents a method for diagnosing faults in systems using FTA to explain the deviations from normal operation observed in sensor outputs. The causes of a system's failure modes will be described in terms of the component states. This will be achieved with the use of coherent and non-coherent fault trees. A coherent fault tree is constructed from AND and OR logic and therefore considers only component-failed states. The non-coherent method expands this, allowing the use of NOT logic, which implies that the existence of component-failed states and component-working states are both taken into account. This paper illustrates the concepts of this method by applying the technique to a simplified water tank level control system.
\end{abstract}

Keywords: fault diagnosis, fault tree analysis

\section{INTRODUCTION}

A system can be analysed for faults in two different ways. The first is to test the system functionality at one point in time. The second continuously monitors the system and detects faults as they occur.

An example of an approach that carries out a series of tests to determine the system status at one point in time is the sequential fault diagnostic tool developed by Novak and co-workers [1-4].

*Corresponding author: Department of Aeronautical and Automotive Engineering, University of Loughborough, Loughborough, Leicestershire LE11 3TU, UK. email: J.D.Andrews@ lboro.ac.uk
The approach uses information about which symptoms are exhibited when the faults are present. The sequential fault diagnostic tool determines the best sequence to conduct the test to locate the fault condition in the cheapest (or quickest) way. A similar method by Pattipati and Alexandridis [5] uses heuristic algorithms in order to determine the most cost-effective sequence of tests. These methods are limited to situations where only a single fault is expected to exist at any point in time; they do not take into consideration multiple component failures. Shakeri et al. [6] extended sequential test sequencing to diagnose multiple failures in systems. However, this method takes a considerable length of time to obtain a diagnosis. 
Another approach to diagnosis is the use of graphical models to describe the propagation of faults in systems. Rao [7] developed two algorithms that use the information from directed graphs of systems to diagnose single failures at one point in time. This technique was developed further by Pattipati [8] to diagnose multiple failures in systems. This graphical method, however, does not take into consideration faults that do not immediately affect the status of the system when they occur.

Failure modes effects analysis (FMEA) is a structured qualitative analysis of a system, subsystem, or function that can be used to identify potential system failure modes, their causes, and the effects on the system operation associated with the failure modes occurrence. Price [9] demonstrated the use of automated FMEA to generate reports that could be used in a diagnostic tool to diagnose multiple faults in systems at one point in time. The failures from the FMEA are only generated to a chosen likelihood of occurrence; therefore all possible outcomes of failure for a system scenario may not be obtained. Paasch and Mocko [10] use FMEA and fault tree analysis (FTA) to develop a model that uses matrix manipulation for diagnosing faults in systems at one point in time. This research does not, however, consider multiple failures.

Papadopoulos [11] has carried out work using state charts and fault trees to provide continuous on-line monitoring and rectification of systems. NOT logic is excluded from the fault trees; therefore only component failures are taken into account to obtain diagnosis. As a result, some faults occurring simultaneously have required conflicting remedial procedures. Yangping et al. [12] also developed a fault-tree-based method that considers only component failures, which uses genetic algorithms to monitor continuously for faults in nuclear power plants. Genetic search is slow in obtaining solutions and there can be problems determining when a global rather than a local diagnosis has been obtained.

Many system failures are not usually the result of one single fault. Therefore the ability to diagnose multiple faults is vitally important. Methods of finding faults or combinations of faults as they occur are the subject of this paper. The approach is based on the fault tree method [13-15]. This is traditionally used to quantify the likelihood of a system failure. In this application the logic diagram is used to develop causes of a system symptom exhibited by a sensor reading, in terms of component conditions. In order to illustrate the features of the method described in this paper it will be applied to a simplified water tank level control system.

\section{THE WATER TANK SYSTEM}

The water tank system is illustrated in Fig. 1. It aims to maintain the level of water between two predetermined limits. In normal operational mode, water flows out of the system through valve V2. The level control system determines when water is flowing from the tank and then refills it by opening valve V1 until the desired tank level is reached. The overspill tray located beneath the tank catches water if

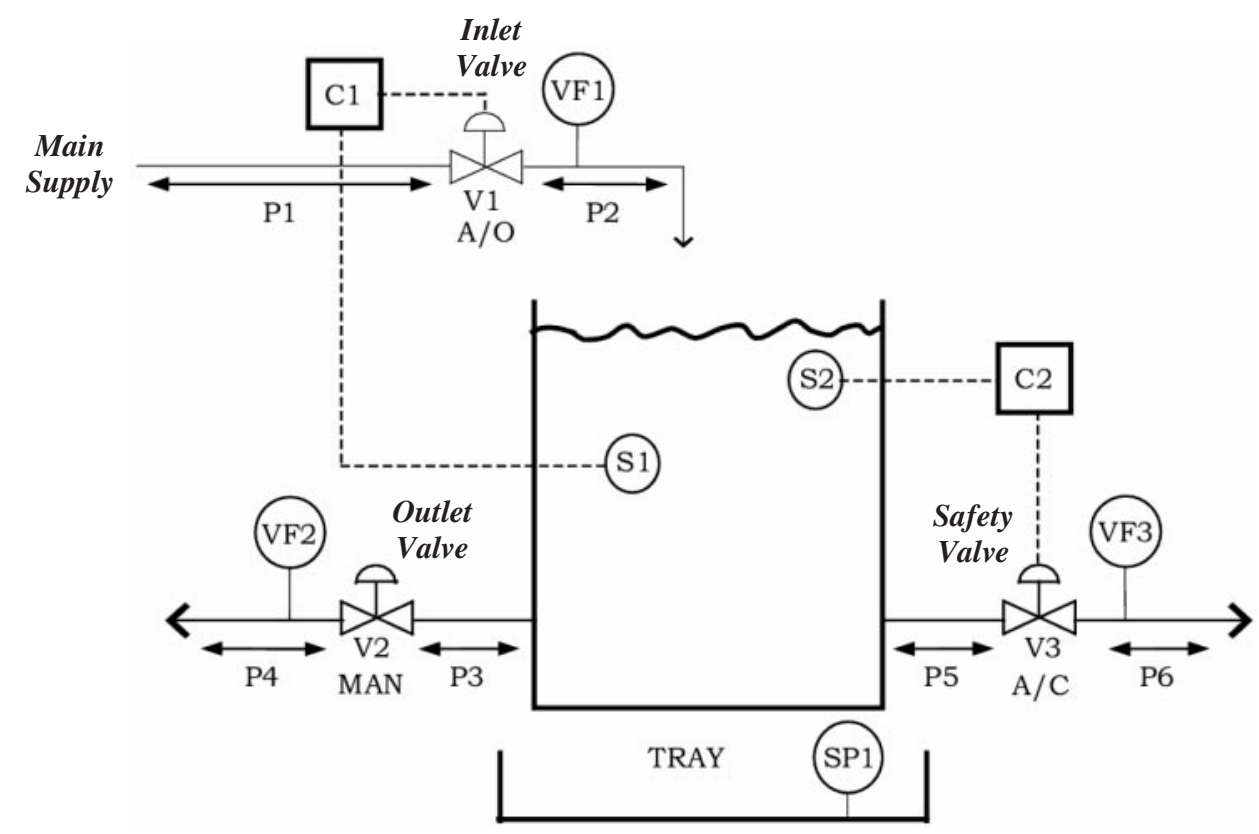

Fig. 1 Water tank system 
the tank has ruptured, if water has leaked out through a crack or hole, or if the water level overflows from the top.

\subsection{System component description}

The water tank system shown in Fig. 1 consists of three valves labelled V1, V2, and V3, two level sensors represented by $\mathrm{S} 1$ and $\mathrm{S} 2$, two controllers $\mathrm{C} 1$ and $\mathrm{C} 2$, and an overspill tray labelled TRAY. There are six sections of pipes identified by the labels P1 to P6.

$\mathrm{V} 1$ is an air-to-open $(\mathrm{A} / \mathrm{O})$ inlet valve controlled by $\mathrm{C} 1$. The level sensor S1 detects the height of the water in the tank. In normal operating mode, if the water in the tank falls below the required level (as indicated by the sensor S1), the controller $\mathrm{C} 1$ would open valve V1, allowing water into the tank. Conversely, if the water in the tank rises to the required level, then $\mathrm{C} 1$ will close V1.

V2 is a manual (MAN) valve, controlled by an operator in response to demand. Finally valve V3 is an air-to-close $(\mathrm{A} / \mathrm{C})$ valve that operates as a safety valve controlled by C2. Normally this will only become operational when a component failure occurs which causes a very high level of water in the tank. A signal from S2 would cause the controller C2 to open valve V3 to reduce the level of water in the tank.

The overspill tray, located underneath the tank, collects any spillages that may occur owing to a failure in the system. So, water in the overspill tray will occur if the tank has ruptured, if the tank is leaking, or if the water level overflows from the top of the tank.

\subsection{System operating modes}

\subsubsection{Sensor locations}

The status of the system is determined using measurements provided by flow sensors situated next to each of the three valves in the system. The sensors are denoted by VF1, VF2, and VF3 for locations at V1, V2, and V3 respectively. These each detect the presence or absence of flow of water, which can be denoted as flow F or no flow NF respectively. For this demonstration study it is assumed the sensors are perfectly reliable. A fourth sensor denoted by SP1 is located in the overspill tray to indicate whether any water has escaped from the tank. Its reading is interpreted as water $\mathrm{W}$ or no water NW. The sensor locations described above are called the system observation points. The observation points for V1, V2, V3, and TRAY generated 16 different scenarios that the system potentially could produce. These are listed in Table 1.
Table 1 System scenarios

\begin{tabular}{rllll}
\hline Scenario & V1 & V2 & V3 & TRAY \\
\hline 1 & F & F & F & W \\
2 & F & F & F & NW \\
3 & F & F & NF & W \\
$\mathbf{4}$ & F & F & NF & NW \\
5 & F & NF & F & W \\
6 & F & NF & F & NW \\
7 & F & NF & NF & W \\
8 & F & NF & NF & NW \\
9 & NF & F & F & W \\
10 & NF & F & F & NW \\
11 & NF & F & NF & W \\
12 & NF & F & NF & NW \\
13 & NF & NF & F & W \\
14 & NF & NF & F & NW \\
15 & NF & NF & NF & W \\
$\mathbf{1 6}$ & NF & NF & NF & NW \\
\hline
\end{tabular}

Were this a real system, additional sensors could be added in order to provide a more complete picture of its operating state. The level control sensors S1 and S2 could also be used. However for the purposes of demonstrating the fault-tree-based fault diagnostics technique the system sensors as described are sufficient, without any additional complexity.

The system has two operating modes; these being ACTIVE when the operator opens valve V2, or DORMANT when V2 is closed. In the ACTIVE operating mode, water is taken out of the system through valve $\mathrm{V} 2$ and the tank is refilled by water coming in through valve V1 from the main water supply. Water would not exit the system through valve V3 and there would be no water in the overspill tray. The sensor readings for the system when ACTIVE should be as those given in scenario 4 in Table 1 . In the DORMANT operating mode the system is effectively on standby with all three valves remaining closed and the overspill tray empty. This should result in the sensor readings given in scenario 16 in Table 1.

The sensor readings given by scenarios 4 and 16 are those which, under steady state conditions, represent the model of how the system should behave when ACTIVE and DORMANT respectively. Given that the system is in the ACTIVE or DORMANT state, any sensor readings that deviate from those expected are regarded as being indicative of some fault within the system.

\subsubsection{Possible component failures}

In order to apply FTA to a system the faults that could occur for each of the system components need to be defined. Table 2 contains a list of possible component failures and their code.

The two operating modes are also represented in the fault trees. ACTIVE signifies that the operator has attempted to open valve V2. DORMANT is used 
Table 2 Potential component failures

\begin{tabular}{ll}
\hline Code & Component failure \\
\hline $\mathrm{P}_{i} \mathrm{~B}(1 \leqslant i \leqslant 6)$ & Pipe $\mathrm{P}_{i}$ is blocked \\
$\mathrm{P}_{i} \mathrm{~F}(1 \leqslant i \leqslant 6)$ & Pipe $\mathrm{P}_{i}$ is fractured \\
$\mathrm{V}_{i} \mathrm{FC}(1 \leqslant i \leqslant 3)$ & Valve $\mathrm{V}_{i}$ fails closed \\
$\mathrm{V}_{i} \mathrm{FO}(1 \leqslant i \leqslant 3)$ & Valve $\mathrm{V}_{i}$ fails open \\
$\mathrm{S}_{i} \mathrm{FH}(1 \leqslant i \leqslant 2)$ & Sensor $\mathrm{S}_{i}$ fails high \\
$\mathrm{S}_{i} \mathrm{FL}(1 \leqslant i \leqslant 2)$ & Sensor $\mathrm{S}_{i}$ fails low \\
$\mathrm{C}_{i} \mathrm{FH}(1 \leqslant i \leqslant 2)$ & Controller $\mathrm{C}_{i}$ fails high \\
$\mathrm{C}_{i} \mathrm{FL}(1 \leqslant i \leqslant 2)$ & Controller $\mathrm{C}_{i}$ fails low \\
$\mathrm{TR}$ & Water tank ruptured \\
$\mathrm{TL}$ & Water tank leaks \\
$\mathrm{NWMS}$ & No water from main supply \\
\hline
\end{tabular}

Table 3 Sensor readings

\begin{tabular}{ll}
\hline Abbreviation & Sensor reading \\
\hline FTV1 & Flow through valve V1 \\
FTV2 & Flow through valve V2 \\
FTV3 & Flow through valve V3 \\
NFTV1 & No flow through valve V1 \\
NFTV2 & No flow through valve V2 \\
WOST & Water in the overspill tray \\
\hline
\end{tabular}

to indicate that the operator has tried to close V2. It should be noted that this is a two-mode system and so only one of the variables ACTIVE or DORMANT can be true at any time.

\section{SENSOR DEVIATION MODELS}

In the application of FTA to the system fault diagnostics a series of failure logic diagrams are produced, representing the causes of any sensor readings. These are developed in a fault tree in terms of the component failure conditions of the system operating state. The list of all possible sensor readings for the system is shown in Table 3.

Two other possible sensor readings for the system are 'no flow through valve V3' and 'no water in the overspill tray'. However, these sensor readings would occur under normal operational conditions (i.e. without any failures occurring) in both the ACTIVE and the DORMANT operating states.

\subsection{Fault tree construction}

Fault trees were generated for each sensor reading listed in Table 3 using both coherent and non-coherent methods. Coherent fault trees are constructed from AND and OR logic and feature only component failure events in the causes of sensor status. The non-coherent method expands this, allowing the use of the NOT operator. This implies that both component-failed and component-working states are

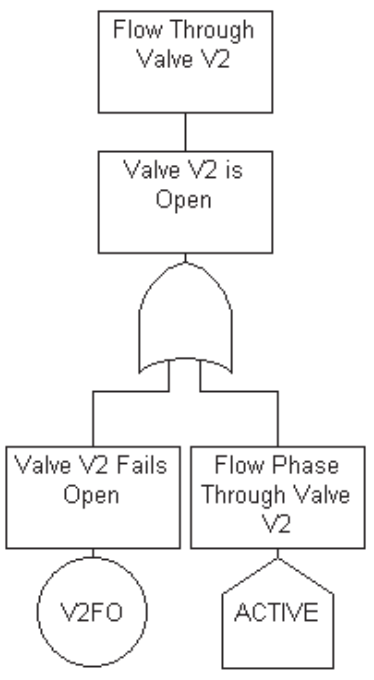

Fig. 2 Coherent fault tree for the sensor reading flow through valve V2

taken into account when developing the causes of the sensor status.

\subsubsection{Coherent fault tree for the sensor reading flow through valve V2}

The coherent fault tree for the sensor reading 'flow through valve V2' is presented in Fig. 2. Referring to Fig. 1, 'flow through valve V2' can only occur because V2 is open. There are two possibilities for having V2 open, these being that either the valve has failed open, which is a basic event, or that the system is in a flow phase and is therefore in the ACTIVE operating mode, represented by a house event. The two prospective outcomes terminate the logic development; in this instance the fault tree is small.

\subsubsection{Non-coherent fault tree for the sensor reading flow through valve V2}

The non-coherent fault tree for the sensor reading 'flow through valve V2' is shown in Figs 3(a) to (c). The information described by the coherent fault tree in section 3.1.1 can now be expanded to include everything in the system that is known not to have failed. Figures 3(a) to (c) show that the introduction of NOT logic significantly increases the amount of information known about the system behaviour.

The sensor reading 'flow through valve V2' occurs because $\mathrm{V} 2$ is open and also because there is water available. As in the coherent fault tree, V2 is open either because it has failed open or because it is in a flow phase (therefore it is in the ACTIVE operating mode). If V2 is open, then it is definitely not closed. Therefore it cannot have failed closed or be in a noflow (DORMANT) phase. 
As well as the valve being open, water has to be available at valve V2 in order to obtain flow. Therefore water can pass through pipes P3 and P4, indicating that P3 is not blocked or fractured and P4 is not blocked. Water also must be available to pass from the tank to pipe P3. Therefore water is contained in the tank, indicating that it has not ruptured, and water is being supplied to the tank, indicating that pipes P1 and P2 are clear and water is coming into the tank. Therefore water is available from the main supply and water can be passed through valve V1. These non-failures together with the potential causes of water through V2 can be combined using an AND gate to form the non-coherent fault tree for the sensor reading.

\subsection{System fault detection}

The system was modelled by four different schemes, of different complexities, in order to construct the scenarios generated from the system observation points (see Table 1). It has been assumed throughout that the system was in a steady state operating condition. Dynamic considerations are also discussed later (see section 4.5).

\subsubsection{Scheme 1}

In this scheme, coherent fault trees were used to develop causes of sensor deviations. At any given time the sensors must indicate one of the 16 scenarios listed in Table 1. If the system is ACTIVE, the sensors should show the readings as in scenario 4 of Table 1. In the DORMANT state they should show the readings in scenario 16 . When the actual readings do not match the pattern of the model, it suggests that a fault exists in at least one of the system components. To find all possible causes, a top event structure can be constructed from the information given by the system observation points. In this scheme, only the observation points giving sensor readings that deviate from the normal operating mode of the system are considered. For a given set of sensor readings the causes of the scenario are developed as fault trees. These are constructed by combining the causes of sensor readings which deviate from that expected using an AND gate. This scheme is now demonstrated assuming that the system is ACTIVE and that the sensor readings from scenario 1 from Table 1 are obtained.

Table 4 contains sensor readings expected when the system is in the ACTIVE operating mode (scenario 4) and the actual reading scenario 1 . The table shows that the sensor reading for valve V3 is 'flow', when in the ACTIVE operating mode it should be 'no flow'. Also the sensor SP1 in the overspill tray should read 'no water' but it is indicating that there is water present. Therefore these sensors show that in scenario 1 there are two sensors that have deviated from their expected readings in the normal operating mode.

In scheme 1 , coherent fault trees for the deviated sensor readings are combined as inputs to an AND gate to form the scenario's top event structure. This is shown in Fig. 4.

A qualitative analysis of this fault tree will produce its minimal cut sets. These are a list of all the combinations of component failures that would cause the sensor readings obtained. Analysis of the fault tree structure given in Fig. 4 produces in total 102 potential causes. From closer inspection, however, only 15 of these will produce the top event structure. The remaining 87 are incorrect; the causes of this are discussed below. The 15 valid potential causes are listed in Table 5.

The coherent fault trees for the sensor readings 'flow through valve V3' and 'water in the overspill

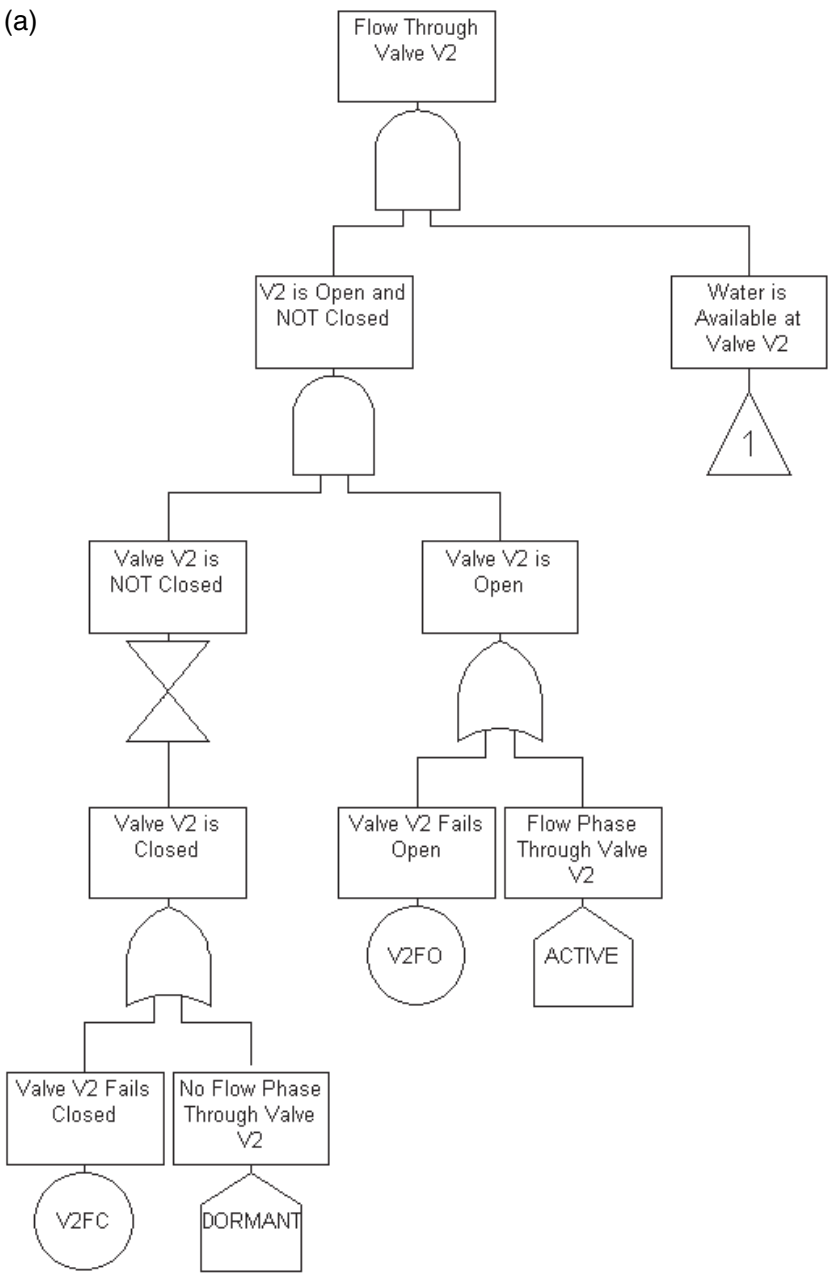

Fig. 3 Non-coherent fault tree for the sensor reading (a) flow through valve V2, (b) flow through valve V2, transfer 1, and (c) flow through valve V2, transfer 2 


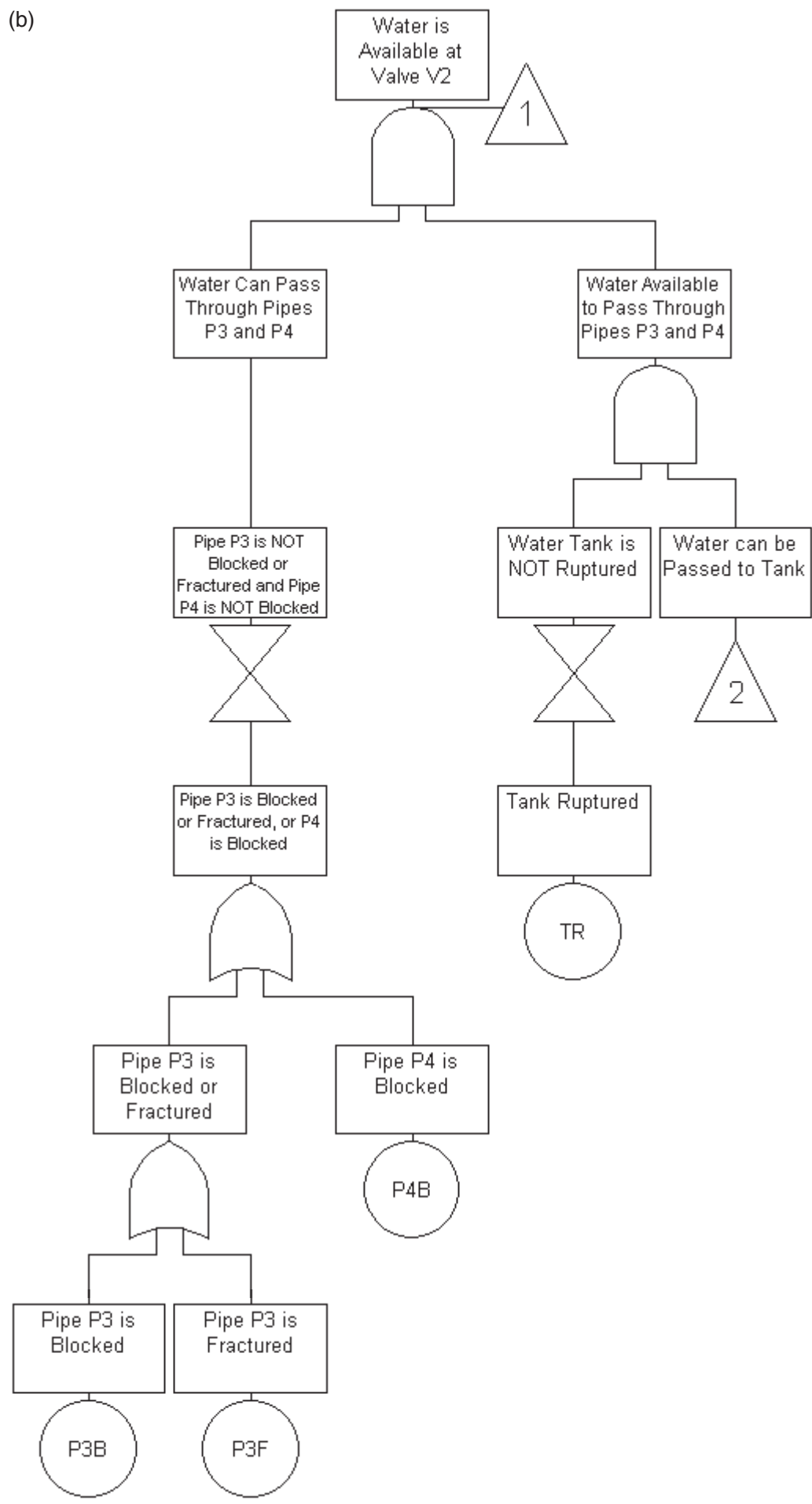

Fig. 3 (Continued)

tray' are both valid when analysed individually. However, one of the reasons for obtaining 'water in the overspill tray' is that no flow through valve V3 occurs, contradicting the sensor reading 'flow through valve V3'. With no NOT logic present in the fault trees to indicate the component working states, conflicting potential causes are not removed. For example, one of the incorrect minimal cut sets is V1FO.P3B.P5B. This minimal cut set would give water in the overspill tray; however, pipe P5 is blocked and so water cannot get through V3, contradicting the top event structure. Therefore coherent fault trees do not contain sufficient information about system behaviour, 
(c)

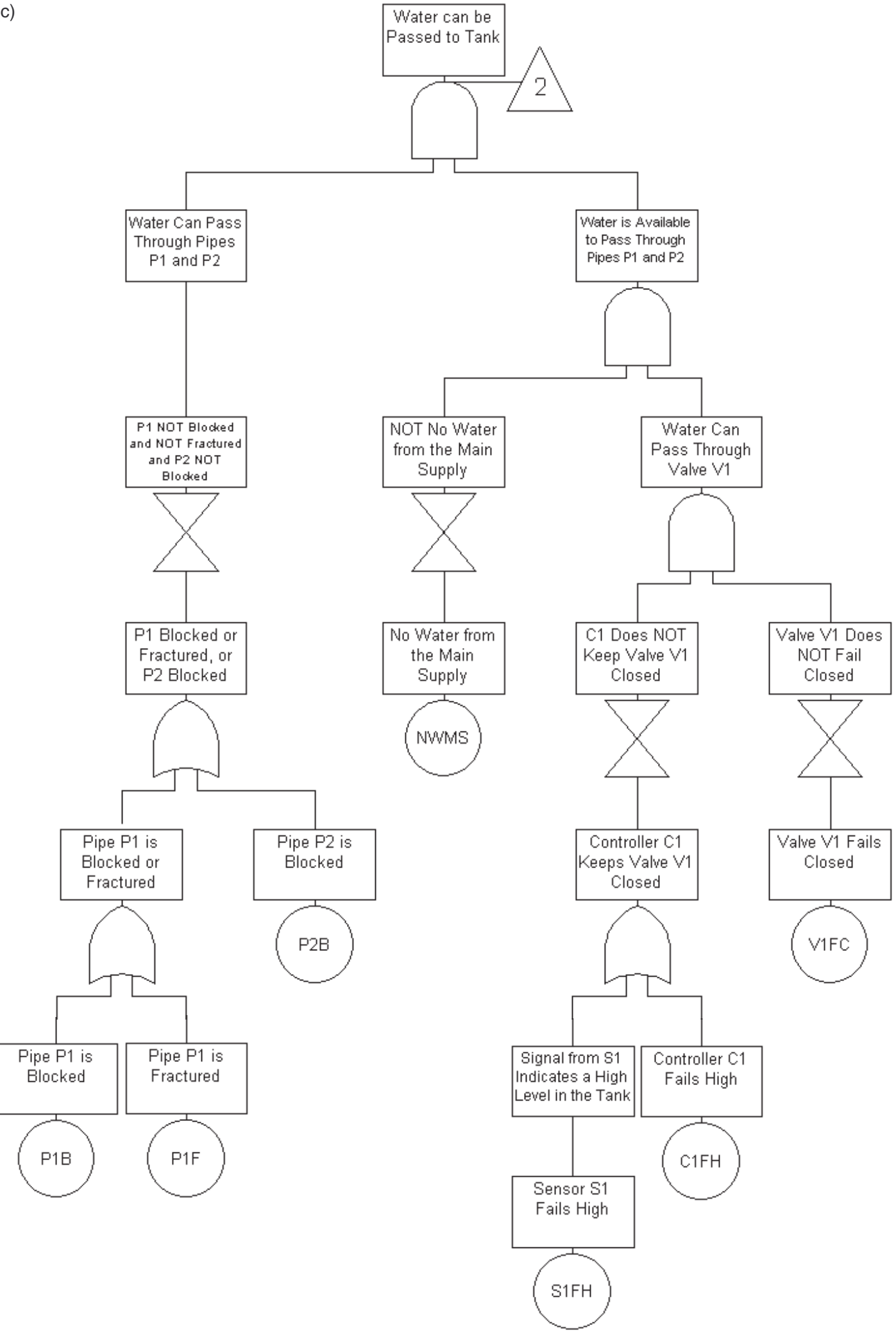

Fig. 3 (Continued)

implying that scheme 1 is not a very reliable method.

As well as the problem of invalid potential causes the number of valid potential causes of scenario 1 listed above is quite large. Therefore it would be difficult to determine which could be the actual cause. Scheme 1 does not take into account the sensor readings from the observation points in the scenario 
that are correct for the chosen operating mode. Only the first three potential causes actually produce scenario 1 when the sensor readings from the observation points at valves $\mathrm{V} 1$ and $\mathrm{V} 2$ are taken into account. The other potential causes are all valid for the sensor reading flow through V1 but would result in no flow through valve V2. This issue is addressed in scheme 2 where information from the sensors that are operating correctly is used to evaluate which parts of the system must function.

\subsubsection{Scheme 2}

Coherent fault trees for sensor readings are also used in scheme 2. In this case, however, a consistency check is introduced into the model by including all sensor readings obtained at any point in the construction of the top event for each scenario. Where sensor readings are as they should be, they are included as NOT failed events in the top event structure. Writing them in their equivalent negated form now includes sensor readings not considered in scheme 1, which are true to the normal chosen operating mode. For example, if the sensor reading for valve V1 were 'flow', then this would be equivalent to saying that there is 'NOT no flow'. This therefore produces a list of working states for flow through valve V1. The fault trees for a given set of sensor readings are constructed by combining both the

Table 4 ACTIVE operating mode with expected and actual sensor readings

\begin{tabular}{lllll}
\hline Scenario & V1 & V2 & V3 & TRAY \\
\hline 4 ACTIVE & F & F & NF & NW \\
1 & F & F & F & W \\
\hline
\end{tabular}

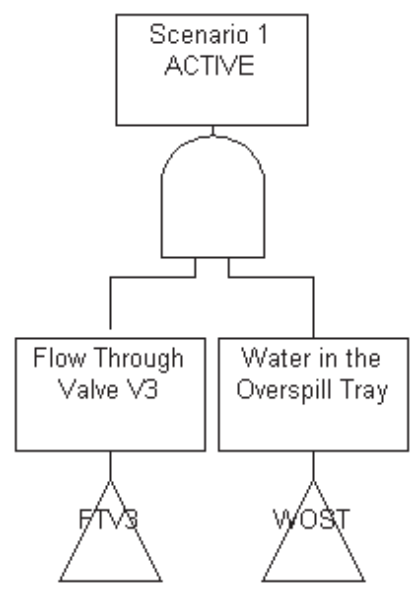

Fig. 4 Top event structure for scenario 1 in the ACTIVE operating mode containing just deviated sensor readings sensor readings that deviate from that expected, together with the expected sensor readings in their equivalent negated form using an AND gate. Scheme 2 is now demonstrated again assuming that the sensor readings of scenario 1 are obtained.

Table 6 contains the ACTIVE operating mode for the system (scenario 4) and scenario 1 with the sensor readings for valves V1 and V2 written in negated form. Fault trees for these sensor readings can now be obtained and included in the model together with those for the deviated sensor readings in order to check consistency.

The coherent fault trees for the deviated sensor readings are combined with the consistency checks as inputs to an AND gate, as shown in Fig. 5 .

A qualitative analysis of this fault tree, which is non-coherent in structure, produces prime implicants [16]. Prime implicants are combinations of component states (working and failed) that produce the sensor readings obtained. As only failed components need to be considered, the working states are then removed from the prime implicants to give the coherent approximation.

The cause of NOT no flow through valve $\mathrm{V} 1$ is $\overline{\mathrm{P} 1 \mathrm{~B}} \cdot \overline{\mathrm{P} 1 \mathrm{~F}} \cdot \overline{\mathrm{P} 2 \mathrm{~B}} \cdot \overline{\mathrm{S} 1 \mathrm{FH}} \cdot \overline{\mathrm{C} 1 \mathrm{FH}} \cdot \overline{\mathrm{V} 1 \mathrm{FC}} \cdot \overline{\mathrm{NWMS}}$

The cause of NOT no flow through valve V2 is

\section{$\overline{\mathrm{TR}} \cdot \overline{\mathrm{NWMS}} \cdot \overline{\mathrm{P} 3 \mathrm{~B}} \cdot \overline{\mathrm{P} 3 \mathrm{~F}} \cdot \overline{\mathrm{P} 4 \mathrm{~B}} \cdot \overline{\mathrm{V} 2 \mathrm{FC}} \cdot \overline{\mathrm{P} 1 \mathrm{~B}} \cdot \overline{\mathrm{P} 1 \mathrm{~F}} \cdot \overline{\mathrm{P} 2 \mathrm{~B}} \cdot \overline{\mathrm{S} 1 \mathrm{FH}}$.} $\overline{\mathrm{C} 1 \mathrm{FH}} \cdot \overline{\mathrm{V} 1 \mathrm{FC}}$

(In the list of component states given above the bar over the event code means NOT that event.)

These combined (ANDed) together with the potential causes listed in Table 5 from scheme 1 shows that scheme 2 produces three potential causes of scenario 1 in the ACTIVE operating mode; these are listed in Table 7 . The potential causes listed

Table 5 Potential causes of scenario 1 in the ACTIVE operating mode using scheme 1

\begin{tabular}{ll}
\hline Number & Potential cause \\
\hline 1 & TL.S2FH \\
2 & TL.C2FH \\
3 & TL.V3FO \\
4 & V1FO.TL.P3B \\
5 & V1FO.TL.P3F \\
6 & V1FO.TL.P4B \\
7 & S1FL.TL.P3B \\
8 & S1FL.TL.P3F \\
9 & S1FL.TL.P4B \\
10 & C1FL.TL.P3B \\
11 & C1FL.TL.P3F \\
12 & C1FL.TL.P4B \\
13 & V1FO.V2FC.TL \\
14 & S1FL.V2FC.TL \\
15 & C1FL.V2FC.TL \\
\hline
\end{tabular}


include the working components. Removing these produces a list of the potential causes containing only failed components, as shown in Table 7 in the column headed Coherent approximation. These are the correct causes of scenario 1. This shows that, for this particular scenario, scheme 2 is a better model at pinpointing the actual cause than scheme 1 is. However, scheme 1 showed that coherent fault trees do not contain sufficient information about system behaviour to obtain a reliable list of potential causes. Scheme 2 uses the same fault trees, and so invalid results from combining the deviated sensor readings may still occur. NOT logic is introduced into the fault trees for each sensor reading in scheme 3 in order to indicate which components are working.

\subsubsection{Scheme 3}

In scheme 3, system behaviour is modelled using non-coherent fault trees. As in scheme 1, the causes of the sensor readings are given in a fault tree structure obtained using only the sensor readings that deviated from the normal system operating mode. Therefore it does not include a consistency check. To illustrate scheme 3, the top event structure (assuming, as before, that the sensor readings represented by scenario 1 have been obtained) can be constructed in the same way as for scheme 1 (see Fig. 4), but using non-coherent fault trees for the deviated sensor readings.

Analysis of the faults, as in scheme 2, produces prime implicants. 15 potential causes of scenario 1 are produced by scheme 3 . After removing the working state events, these are the same as those obtained by scheme 1 which are valid for the deviated sensor readings (see Table 5). Scheme 3 therefore shows that introducing NOT logic into the fault trees indicates which components are working for each deviated sensor reading and removes any conflicting potential causes.

Table 6 ACTIVE operating mode and scenario 1

\begin{tabular}{lllll}
\hline Scenario & V1 & V2 & V3 & TRAY \\
\hline 4 ACTIVE & F & F & NF & NW \\
1 & $\overline{N F}$ & $\overline{N F}$ & $\mathbf{F}$ & $\mathbf{W}$ \\
\hline
\end{tabular}

There are again quite a large number of alternatives and it would be difficult to determine which could be the actual cause. In the same way as for scheme 1, scheme 3 does not take into account the sensor readings from the observation points in the scenario that are correct for the chosen operating mode. Some of the potential causes listed in the example may be invalid. This scheme has failed to differentiate between the potential causes and so scheme 4 , which contains the additional complexity of non-coherent sensor deviations with consistency checks, may provide a better analysis option.

\subsubsection{Scheme 4}

Scheme 4 modelled system behaviour using noncoherent fault trees for sensor deviations. The top event structure for scenario 1 using scheme 4 is shown in Fig. 5. Analysis of the faults, as in schemes 2 and 3, produces prime implicants. Removing the working component states gives the coherent approximation. There are three potential causes of

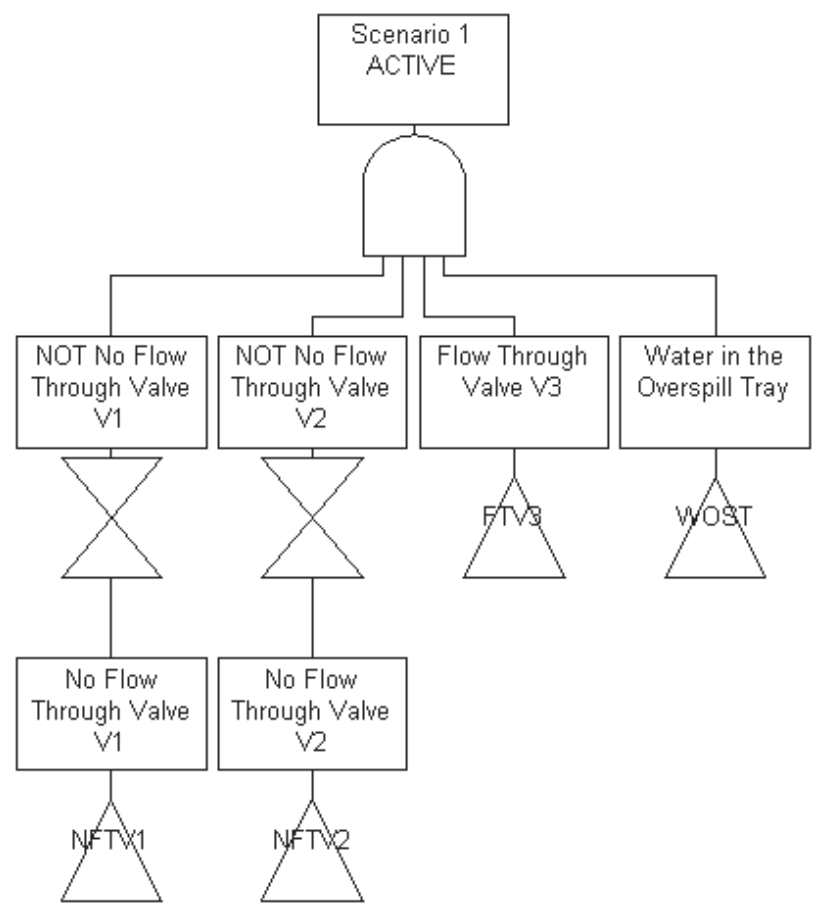

Fig. 5 Top event structure for scenario 1 in the ACTIVE operating mode containing both deviated sensor readings and consistency checks

Table 7 Potential causes of scenario 1 in the ACTIVE operating mode using scheme 2

\begin{tabular}{|c|c|c|}
\hline Number & Potential cause & $\begin{array}{l}\text { Coherent } \\
\text { approximation }\end{array}$ \\
\hline 1 & $\overline{\mathrm{NWMS}} \cdot \overline{\mathrm{TR}} \cdot \overline{\mathrm{P} 2 \mathrm{~B}} \cdot \overline{\mathrm{P} 1 \mathrm{~F}} \cdot \overline{\mathrm{P} 1 \mathrm{~B}} \cdot \overline{\mathrm{V} 2 \mathrm{FC}} \cdot \mathrm{TL} \cdot \overline{\mathrm{P} 4 \mathrm{~B}} \cdot \overline{\mathrm{P} 3 \mathrm{~B}} \cdot \overline{\mathrm{V} 1 \mathrm{FC}} \cdot \overline{\mathrm{C} 1 \mathrm{FH}} \cdot \overline{\mathrm{S} 1 \mathrm{FH}} \cdot \mathrm{S} 2 \mathrm{FH}$ & TL.S2FH \\
\hline 2 & NWMS.TR.P2B.P1F.P1B.V2FC.TL.P4B.P3B.V1FC.C1FH.S1FH.C2FH & TL.C2FH \\
\hline 3 & NWMS.$\overline{T R} \cdot \overline{\mathrm{P} 2 \mathrm{~B}} \cdot \overline{\mathrm{P} 1 \mathrm{~F}} \cdot \overline{\mathrm{P} 1 \mathrm{~B}} \cdot \overline{\mathrm{V} 2 \mathrm{FC}} \cdot \mathrm{TL} \cdot \overline{\mathrm{P} 4 \mathrm{~B}} \cdot \overline{\mathrm{P}} \mathrm{B} \cdot \overline{\mathrm{V} 1 \mathrm{FC}} \cdot \overline{\mathrm{C} 1 \mathrm{FH}} \cdot \overline{\mathrm{S} 1 \mathrm{FH}} \cdot \mathrm{V3FO}$ & TL.V3FO \\
\hline
\end{tabular}


Table 8 Potential causes of scenario 1 in the ACTIVE operating mode using scheme 4

\begin{tabular}{ll}
\hline Number & Potential causes \\
\hline 1 & TL.S2FH \\
2 & TL.C2FH \\
3 & TL.V3FO \\
\hline
\end{tabular}

scenario 1, listed in Table 8 . The potential causes of scenario 1 in Table 8 are the same as those obtained using scheme 2 after removing the working components (see Table 7). This shows that schemes 2 and 4 are better models than schemes 1 and 3 at pinpointing the actual cause of scenario 1. Compared with the result obtained from scheme 3 , the model in scheme 4 strengthens the belief that not sufficient information is being obtained by considering only sensors that have deviated from their expected readings.

The performances of the four schemes have been investigated to evaluate their potential causes of all 16 scenarios. Schemes 1 and 2 have both produced potential causes that are invalid to the top event structure. For example, for scenario 5, schemes 1 and 2 produce 132 and 111 potential causes respectively for their top event structures. Only 24 of these results for the two schemes are actually valid even though the fault trees for the sensor readings are correct when analysed individually. For certain scenarios, scheme 2 has also removed potential causes that are valid to the top event structure. This implies that there is not sufficient information in the coherent fault trees to explain the system behaviour. Scheme 4 produces the best performance for both the ACTIVE and the DORMANT operating modes.

\subsection{Switching the system operating modes}

The two operating modes for the system are ACTIVE when the operator opens valve V2, or DORMANT when V2 is closed. The operating state of the system can be changed in order to examine the system behaviour. This can be used alongside any of the other four schemes previously discussed. Schemes 2 and 4 have both narrowed the potential causes of scenario 1 in the ACTIVE operating mode down to three possibilities, (see Table 8). These all indicate that the tank is definitely leaking (TL) to create water in the overspill tray, but it is unclear as to whether sensor S2 has failed high (S2FH), controller $\mathrm{C} 2$ has failed high $(\mathrm{C} 2 \mathrm{FH})$, or valve $\mathrm{V} 3$ has failed open (V3FO) in order to cause flow through valve V3.

If the real cause were, for example, TL.S2FH (the first potential cause listed in Table 8), one way to narrow down the possibilities would be to switch the operating mode of the system, in this case from ACTIVE to DORMANT. Switching operating modes could also highlight hidden potential failures for valve V2. For example, if V2 failed open while the system was working as in scenario 1 in the ACTIVE operating mode, then this failure would be masked. Switching to the DORMANT operating mode would reveal this failure, which would otherwise have remained undetected. For scenarios where V2 has already failed in a given operating mode, switching modes could conceal this failure. For example, if there is flow through valves $\mathrm{V} 1$ and $\mathrm{V} 2$, no flow through V3, and water in the overspill tray in the DORMANT operating mode and the system is switched to the ACTIVE mode, then the failure at V2 would become hidden.

In the DORMANT mode a different set of sensor readings resulting from TL.S2FH would occur, which will be one of two possibilities depending on whether or not V2 was found to be working correctly. The sensors in the ACTIVE operating mode indicate that there is flow through all three valves and water in the overspill tray. When the operating mode is switched to DORMANT, there should be no flow through V2. If this is the case, then the new set of deviated sensor readings will be no flow through V2, flow through V1 and V3, and water in the overspill tray. If there is still flow through valve V2 after switching operating modes, then this indicates that all four sensors have deviated from their normal operating modes. Treating this set of sensor values by comparison with the expected set, namely scenario 16 in Table 1, and applying any of the schemes 1 to 4 will produce a set of possible causes. The true cause of the fault will be in both lists. Valve V2 failing if not apparent before switching modes will be indicated by the addition of this component failure to the potential causes in the list from the mode that the system has been switched to.

\section{DISCUSSION}

\subsection{Effectiveness of the schemes}

Schemes 1 to 4 were applied to the 16 scenarios of possible sensor readings listed in Table 1. An index has been applied to each of the schemes to give some indication of their relative merits. For each scheme the number of possible causes indicated is reported, together with how many of the correct causes are included in this list.

An index to indicate the effectiveness of the scheme is calculated using the ratio of the number of valid causes to the total number of potential causes and averaged over the scenarios. The index 
is used on each scheme to indicate how effective it has been at obtaining correct potential causes of failure over the 15 scenarios that contain at least one deviated sensor reading (one of the scenarios represents the symptoms of a fully functioning system).

\subsubsection{Definition of effectiveness index}

The effectiveness index $I_{\mathrm{E}}$ used is defined as

$$
I_{\mathrm{E}}=\frac{1}{N} \sum_{i=1}^{N} \frac{n c_{i}}{n_{i}}
$$

where

$$
\begin{gathered}
N=\text { number of scenarios investigated } \\
n_{i}=\text { number of potential causes of failure identi- } \\
\text { fied by the scheme for scenario } i \\
n c_{i}=\text { number of correct potential causes of failure } \\
\text { obtained by the scheme for scenario } i
\end{gathered}
$$

\subsubsection{Results}

The DORMANT mode is the less interesting situation for the tank system and so the discussion is focused on the ACTIVE system operation. Results obtained for schemes 1 to 4 in the ACTIVE operating mode and their respective effectiveness indices are shown in Table 9. For each scheme and each scenario the table contains the number of possible causes produced by the scheme and the number of these that are correct causes of the symptoms observed. The actual number of causes that would have been produced if the scheme gave a perfectly correct list is indicated in the final column of the table.

The validity of any potential cause of the observed symptoms has been checked regarding its immediate effects on the system. Situations are not considered where the symptoms change in time, e.g. longer term if the water level is increasing and the safety valve V3 opens; there is initially no flow through V3 which changes when the valve opens. Dynamic effects are not investigated here. The effectiveness index is given for each scheme.

Schemes 1 and 2 have both produced potential causes that are invalid to the top event structure, even though the fault trees for the sensor readings are correct when analysed individually. This implies that coherent fault trees do not contain sufficient information about the system to produce an accurate list of potential causes. When the working states are considered as in scheme 2 , the effectiveness is improved, as indicated by the index increasing from 0.296 to 0.750 . Scheme 4 uses non-coherent fault trees and information from all sensors; therefore the causes obtained are more accurate than those obtained by schemes 1 to 3 and this is reflected in the $I_{\mathrm{E}}$ result.

\subsection{Scalability and consistency checks}

The methods presented in this paper for diagnosing faults in systems using FTA to explain the deviations from normal values observed in the sensed variables have been illustrated using a simple example containing only a small number of components. Application to a larger system is required in order to show how effective the methods would be when used on real systems.

The computational expense from a large number of sensors is the main problem. Introducing consis-

\begin{tabular}{|c|c|c|c|c|c|c|c|c|c|c|c|c|c|}
\hline \multirow[b]{2}{*}{$\underline{\text { Scenario }}$} & \multirow[b]{2}{*}{$\mathrm{V} 1$} & \multirow[b]{2}{*}{$\mathrm{V} 2$} & \multirow[b]{2}{*}{ V3 } & \multirow[b]{2}{*}{ TRAY } & \multicolumn{2}{|c|}{ Scheme 1} & \multicolumn{2}{|c|}{ Scheme 2} & \multicolumn{2}{|c|}{ Scheme 3} & \multicolumn{2}{|c|}{ Scheme 4} & \multirow{2}{*}{$\begin{array}{l}\text { Number of actual } \\
\text { possibilities }\end{array}$} \\
\hline & & & & & $n_{i}$ & $n c_{i}$ & $n_{i}$ & $n c_{i}$ & $n_{i}$ & $n c_{i}$ & $n_{i}$ & $n c_{i}$ & \\
\hline 1 & $\mathrm{~F}$ & $\mathrm{~F}$ & $\mathrm{~F}$ & W & 102 & 3 & 3 & 3 & 15 & 3 & 3 & 3 & 3 \\
\hline 2 & $\mathrm{~F}$ & $\mathrm{~F}$ & $\mathrm{~F}$ & NW & 15 & 3 & 3 & 3 & 15 & 3 & 3 & 3 & 3 \\
\hline 3 & $\mathrm{~F}$ & $\mathrm{~F}$ & $\mathrm{NF}$ & W & 74 & 1 & 1 & 1 & 74 & 1 & 1 & 1 & 1 \\
\hline 4 & $\mathbf{F}$ & $\mathbf{F}$ & NF & NW & - & - & - & - & - & - & - & - & - \\
\hline 5 & $\mathrm{~F}$ & NF & $\mathrm{F}$ & W & 132 & 24 & 111 & 24 & 45 & 24 & 24 & 24 & 24 \\
\hline 6 & $\mathrm{~F}$ & NF & $\mathrm{F}$ & NW & 48 & 24 & 24 & 24 & 45 & 24 & 24 & 24 & 24 \\
\hline 7 & $\mathrm{~F}$ & NF & NF & W & 84 & 77 & 77 & 77 & 84 & 77 & 77 & 77 & 77 \\
\hline 8 & $\mathrm{~F}$ & $\mathrm{NF}$ & $\mathrm{NF}$ & NW & 12 & 4 & 4 & 4 & 12 & 4 & 4 & 4 & 4 \\
\hline 9 & $\mathrm{NF}$ & $\mathrm{F}$ & $\mathrm{F}$ & W & 714 & 21 & 21 & 21 & 21 & 21 & 21 & 21 & 21 \\
\hline 10 & NF & $\mathrm{F}$ & $\mathrm{F}$ & NW & 105 & 21 & 21 & 21 & 21 & 21 & 21 & 21 & 21 \\
\hline 11 & $\mathrm{NF}$ & $\mathrm{F}$ & $\mathrm{NF}$ & W & 518 & 7 & 7 & 7 & 14 & 7 & 7 & 7 & 7 \\
\hline 12 & $\mathrm{NF}$ & $\mathrm{F}$ & $\mathrm{NF}$ & NW & 7 & 7 & 7 & 7 & 7 & 7 & 7 & 7 & 7 \\
\hline 13 & $\mathrm{NF}$ & NF & $\mathrm{F}$ & W & 714 & 0 & 714 & 0 & 21 & 0 & 21 & 0 & 84 \\
\hline 14 & NF & $\mathrm{NF}$ & $\mathrm{F}$ & NW & 105 & 0 & 105 & 0 & 21 & 0 & 21 & 0 & 84 \\
\hline 15 & $\mathrm{NF}$ & NF & NF & W & 518 & 14 & 518 & 14 & 14 & 14 & 14 & 14 & 14 \\
\hline \multicolumn{5}{|c|}{ Effectiveness index $I_{\mathrm{E}}$} & \multicolumn{2}{|c|}{0.296} & \multicolumn{2}{|c|}{0.750} & \multicolumn{2}{|c|}{0.549} & \multicolumn{2}{|c|}{0.867} & \\
\hline
\end{tabular}
tency checks may be prohibitive for real-time analysis of industrial systems. Scheme 4 could be heavy on computer processing power, but scheme 3 is not as reliable because it does not check consistency.

Table 9 Results obtained for schemes 1 to 4 in the ACTIVE operating mode and effectiveness index 
However, this can be solved by taking the potential causes obtained from just the deviated sensor readings and checking these individually against those from the sensor readings which are true to the operating mode. Any potential causes that lead to a contradiction can then be removed from the list.

\subsection{Imperfect sensors}

The method illustrated in this paper has been applied to an example that does not account for the possibility that there are imperfect sensors in the system. The example used demonstrates the approach taken to fault detection. It could accommodate sensor failures by incorporating these events into the fault trees for each sensor reading and the analysis performed in the same way.

\subsection{Obtaining the exact cause of failure}

Ideally the most efficient scheme to model the behaviour of a system would be one that could determine the exact cause of each scenario. However, in reality there may be more than one possible cause. For example, there are three potential causes of scenario 1 in the ACTIVE operating mode (see Table 8). These all indicate that the tank is definitely leaking (TL) to create water in the overspill tray, but it is unclear as to whether sensor S2 has failed high (S2FH), controller $\mathrm{C} 2$ has failed high $(\mathrm{C} 2 \mathrm{FH})$, or valve V3 has failed open (V3FO) in order to cause flow through valve V3.

Valve V3 can fail in three different ways that would still produce the same outcome for the top event structure. Switching the operating mode in this case does not reduce the number of potential causes but could highlight whether there is a problem at valve V2. Therefore, a probability for each component would have to be introduced in order to deduce which is most likely to have caused the scenario. This addition to the calculation procedure would effectively be providing measures $[\mathbf{1 3}]$ for each minimal cut set.

\subsection{Dynamic effects}

At present the water tank system is observed in a steady state. Because of this it is not possible, without taking into account transient effects, for some of the 16 scenarios to occur. For example there are scenarios containing a 'no flow' sensor reading for valve V1 and flow through at least one of the other two valves produced no potential causes, indicating that they could never happen. However, if dynamics were taken into consideration, then depending on the state of the system these scenarios may occur, albeit for a short period of time. Therefore considering the effects of dynamics on the water tank would provide a more realistic model of the system behaviour.

\subsection{Two-phase system}

The water tank system is assumed to be in one of two phases, these being the flow phase through valve V2 when in the ACTIVE operating mode and the no-flow phase through V2 in the DORMANT operating mode. However, V2 could be partially open, indicating an intermediate flow phase through the valve. Further analysis could therefore consider the behaviour of the system when failure modes are not clear.

\section{CONCLUSIONS}

1. FTA can be used to identify multiple faults in a systems fault diagnostic capability.

2. Non-coherent fault trees to represent the causes of sensor outputs provide more reliable results than those obtained using coherent fault trees.

3. The introduction of consistency checks with the information provided by all sensors is required in order to produce an accurate list of all potential causes of the current system state.

4. Scheme 4, which uses non-coherent fault trees and checks for consistency by using information from all the observation points, is the most accurate of schemes 1 to 4 for modelling the behaviour of the water tank system.

5. Switching the operating mode of the system can be used alongside scheme 4 to produce a more precise list of potential causes should the system function enable mode switching.

\section{ACKNOWLEDGEMENT}

The research reported in this paper has been carried out with financial support from BAE Systems.

\section{REFERENCES}

1 Zuzek, A., Biasizzo, A., and Novak, F. Towards a general test presentation in the test sequencing problem. In Proceedings of the Second International On-Line Testing Workshop, (New York). IEEE Computer Society Press, Biarritz, France, 1996, pp. 236-237.

2 Zuzek, A., Novak, F., Biasizzo, A., Savnik, I., and Cestnik, B. Sequential diagnosis tool for system maintenance and repair. Electrotech. Rev., 1995, 62 224-231.

3 Biasizzo, A., Zuzek, A., and Novak, F. Sequential diagnosis tool. Microprocessors Microsystems, 2000, 24 191-197. 
4 Biasizzo, A., Zuzek, A., and Novak, F. Sequential diagnosis with asymmetrical tests. Computer J., 1998, 41(3) 163-170.

5 Pattipati, K. R. and Alexandridis, M. G. Application of heuristic search and information theory to sequential fault diagnosis. IEEE Trans. Systems, Man, Cybernetics, 1990, 20(4), 872-887.

6 Shakeri, M., Raghavan, V., Pattipati, K. R., and Patterson-Hine, A. Sequential testing algorithms for multiple fault diagnosis. IEEE Trans. Systems, Man, Cybernetics, Part A: Systems Humans, 2000, 30(1) 1-14.

7 Rao, N. S. V. Expected-value analysis of two single fault diagnosis algorithms. IEEE Trans. Computers, 1993, 42(3) 272-280.

8 Pattipati, K. R. Computationally efficient algorithms for multiple fault diagnosis in large graph-based systems. IEEE Trans. System, Man, Cybernetics, Part A: Systems Humans, 2003, 33(1), 73-85.

9 Price, C. Computer-based diagnostic systems, 1999 (Springer-Verlag, London).

10 Paasch, R. and Mocko, G. Incorporating uncertainty in diagnostic analysis of mechanical systems.
Trans. ASME, J. Mech. Design, 2005, 217(2), 315-325.

11 Papadopoulos, Y. Model-based system monitoring and diagnosis of failures using statecharts and fault trees. Int. J. Reliability Engng System Safety, 2003, 81 325-341.

12 Zhou Yangping, Zhoo Bingquan, and Wu Dong Xiu Application of genetic algorithms to fault diagnosis in nuclear power plants. Reliability Engng System Safety, 2000, 67, 153-160.

13 Andrews, J. D. and Moss, T. R. Reliability and risk assessment, 2nd edition, 2002 (Professional Engineering Publishing Limited, Bury St Edmunds and London).

14 Lee, W. S., Grosh, D. L., Tillman, F. A., and Lie, C. H. Fault tree analysis methods and applications - a review. IEEE Trans. Reliability, 1985, 34(3) 194-203.

15 Billinton, R. and Allan R. N. Reliability evaluation of engineering systems, 2nd edition, 1992 (Plenum, New York).

16 Andrews, J. D. The use of NOT logic in fault tree analysis. Quality Reliability Engng Int., 2001, 17, 143-150. 